\title{
UAV Control Architecture: Review
}

\author{
IDALENE Asmaa ${ }^{1}$, BOUKHDIR Khalid ${ }^{2}$, and MEDROMI Hicham ${ }^{3}$ \\ ${ }^{1,2,3}$ Research and Engineering Laboratory LRI, National School of Electricity and Mechanics Casablanca, Morocco \\ ${ }^{1}$ Pluridisciplinary Laboratory of Research and Innovation (LPRI), EMSI Casablanca, Morocco
}

\begin{abstract}
Since civil Unmanned Aerial Vehicles (UAVs) are expected to perform a wide rang of mission, the subject of designing an efficient control architecture for autonomous UAV is a very challenging problem. Several contributions had been done in order to implement an autonomous UAV. The key challenge of all these contributions is to develop the global strategy. Robotic control approaches could be classified into six categories: Deliberative, Reactive, Hybrid, Behavior, Hybrid Behavior and subsumption approach. In this paper, we will review the existing control architectures to extract the main features of civil UAVs. The definition, advantage and drawback of each architecture will be highlighted to finally provide a comparative study of the mentioned control approaches.
\end{abstract}

Keywords-Unmanned Aerial Vehicle; control architecture; deliberative approach; reactive approach; hybrid approach; behavior approach; hybrid behavior approach; subsumption approach

\section{INTRODUCTION}

The Unmanned aerial vehicle or UAV is a particular mobile robot that operates in dynamic tri-dimensional space [1] [2], in order to meet some goals or to achieve a given mission by executing a specific control strategy [3] [2] . Typically, autonomous UAV is a complex system [4] that adapts to different circumstances without human intervention [5] [6]. Autonomous UAV needs continually to sense and perceive its surrounding environment, to decide what to do and to execute the appropriate commands in its operating environment [7] [8].

UAV control architecture represents a global strategy and specific algorithms used to define how the UAV will perform its sensing operations and its perceiving capabilities [7] [9], how it will decide its task to meet and how it will act in specific environment conditions [10]. The control architecture affects the robot's capabilities: the processing time, the need to completely know the operating environment, the ability to deal with a large variety of missions, the capacity to achieve goals in the presence of uncertainties and the autonomy level [11], [12].

Several contributions have been done in term of control architectures [10] [13] [14] [15] [16]. beyond the known control architectures, we found the deliberative approach that has been implemented based on the sense-plan-act paradigm [17] [18]. The reactive architecture has been designed as a set of condition-action pairs [19] [20]. While the hybrid approach has been made as a combination of deliberative and reactive capabilities [21], [22]. Moreover, the behavior approach has been defined as a collection of behavior sequences that performs each of them a specific goal [23]. And finally we found the subsumption approach that has been structured as a set of ordered competence levels, each of which provides a specific capability [24], [25]. The key challenge for all these contributions is to develop an autonomous control system that can make suitable decisions, perform many tasks, plan a feasible path and avoid static and dynamic obstacles [12].

This paper is organized as follows: in the second section the existing control architectures for mobile robot will be reviewed, a comparative study of the state of the art will be proposed in the third section and finally a brief conclusion of this work will be presented in section five.

\section{CONTRol ARCHitecture: REVIEW}

Various control architectures have been designed in order to develop high performance systems [10], [13], [14], [15], [16]. Each of them offers new concepts in attempt to build an autonomous robot. In the present section we review in detail the existing control architectures:

\section{A. The Deliberative based Control Architecture}

The deliberative control architecture is a top-down approach [13]. In order to meet a given mission, the deliberative approach reasons about goals and constraints to finally execute low-level commands. Basically, it comprises three generic sequential functionalities: sensing, planning and acting modules [17]. For each mission's goal (see Fig. 1), the sensing module senses the robot's surrounding environment in order to update a given world model. To reach the mission goal, the planning module generates a valid task plan considering the robot's constraints. At last, the acting module transforms the task plan into robot low-level commands then executes these commands. The robot then repeats these sequential functionalities until it reaches its mission goals [26].

This architecture presents a serious source of weakness in certain cases, we mention some of them [27], [28]:

- If one of the modules fails, the whole architecture will break.

- It is ineffective in a dynamic or uncertain environment.

- It requires high performance computational capabilities: memory and processing time to build a complete world model.

- It has a higher chance of failing if the representation of the world model is neither exact nor complete.

\section{B. The Reactive based Control Architecture}

Reactive control architecture is a bottom-up approach that was developed in order to hold some drawbacks of the deliberative control architecture [13], [10]. It consists of reactive rules 


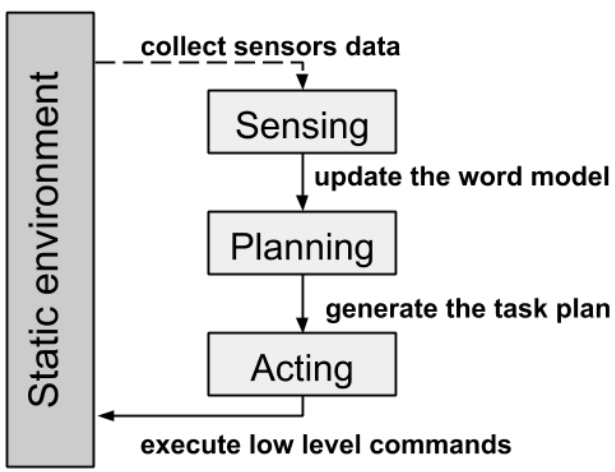

Fig. 1. Deliberative architecture

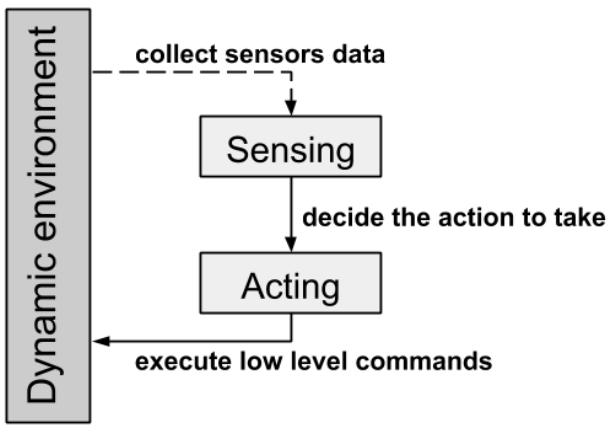

Fig. 2. Reactive architecture

set that deals with environmental changes [29], This architecture implements a control strategy as a collection of conditionaction pairs that couples a sensor data to robot action. It can operate in a dynamic environment; without neither building world model nor performing planning functionality; simply by generating control commands based on sensory information [19].

As it shown in Fig. 2, the robot perceives its surrounding world by gathering its sensor data, then automatically decides what action to take. This architecture is computationally simpler than the deliberative approach and has a faster response to dynamic changes without any prior environment knowledge [10].

However, the reactive based approach cannot deal in a situation with high-level goals and complex constraints [30]. Thereby, it exclusively solves the class of problems in which the environment is well known, the goals are well defined and the robot is equipped with enough sensors that allow it to perceive the necessary information [31].

\section{The Hybrid Control Architecture}

To fulfill a robot mission in a real world, some features of deliberative architecture combined with the reactive architecture are required [13], [10], [32]. The hybrid approach was designed to deal with high-level goals and complex constraints in a dynamic environment [21]. It offers a compromise between reactive and deliberative-based approaches [33]. Typically, the hybrid control architecture (see Fig. 3) employs threehierarchical levels:

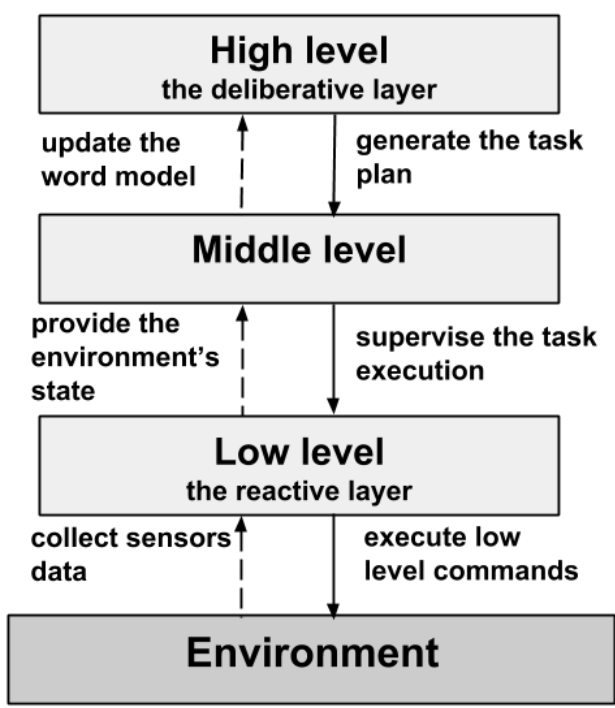

Fig. 3. Hybrid architecture

- $\quad$ High level (the deliberative layer) for decision making. This level performs complex computations to generate a valid task plan that corresponds to a set of actions. Each action defines a specific commands sequence sent to the reactive layer in order to generate the desired action.

- Middle level supervises the interaction between the high level and low level.

- Low level (the reactive layer) for low control senses the environment. It takes care of the immediate safety of the robot such as obstacle avoidance. To generate the robot's motion, low level executes the actions sequence provided by the deliberative layer.

\section{The Behavior Control Architecture}

Behavior-based control architecture [34]; which is inspired by biological studies; is designed to perform a reactive mapping between perception and action modules [35]. Basically, the behavior approach divides the control strategy into a set of behaviors as it shown if Fig. 4. Each of which is responsible for a particular task [36].

This architecture provides some advantages that make this approach more powerful than the reactive one [37], [38]. Each behavior can provide both reactive and deliberative capabilities. This architecture could deal with an unpredictable situation the robot may face without having to know the environment. Furthermore, it offers parallel and concurrently behaviors collection that act independently to achieve the robot goals. Moreover, it provides a good solution for robot's problem carrying out tasks in multiple and unknown environments.

However, this architecture presents some inconvenient:

- To control a robot, this approach must combine and coordinate several behaviors. But in some cases, it's difficult to choose the prior behavior to execute in first.

- Since behaviors represent low-level control, they may not deal with high-level goals. 


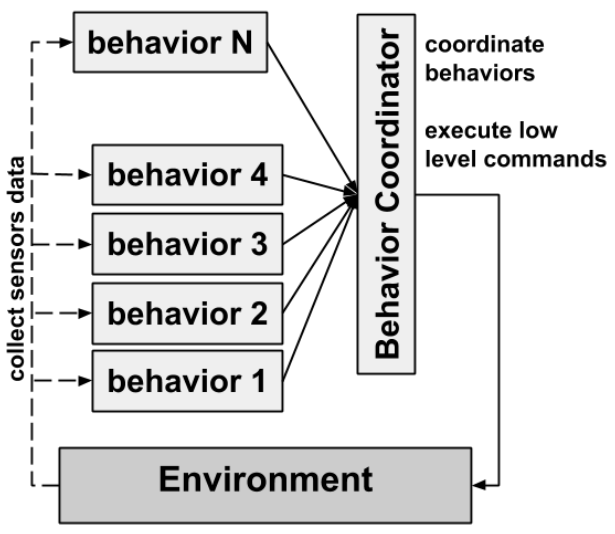

Fig. 4. Behavior architecture

- The lack of the planning module may result in not solving complicated tasks.

\section{E. The Hybrid Behavior Control Architecture}

The main purpose of the hybrid behavior control architecture is to resolve some limits of both the hybrid and the behavior architecture [39] [40] [41]:

- It can deal with high-level goals by generating the appropriate task plan.

- It coordinates the existing behavior to reach the mission objectives.

- It is designed to be flexible. layers:

The hybrid behavior control architecture includes three

High level (called cognitive layer) reasons about mission goals and constraints. It makes task-oriented reasoning and planning, and it decides how to coordinate the current behaviors.

Middle level (or behavior layer) defines all robot's behaviors. Each of which performs a specific task.

Low level was designed to achieve the best performance of each individual behavior and to execute the low commands.

In view of inconvenient of the hybrid behavior approach we can see:

- The same architecture cannot be reused on various robot types.

- This architecture suffers from layers interdependency.

\section{F. The Subsumption Architecture}

The Subsumption architecture [24] was designed in order to offer a feasible approach for building a robust robot system. It provides a good solution for control problem dealing with multiple sensors and goals. This approach proposes an extensible layered architecture and provides a unified representation of large scale of robots [42].

The Subsumption approach divides the control problem into a set of ordered layers according to the task achieving

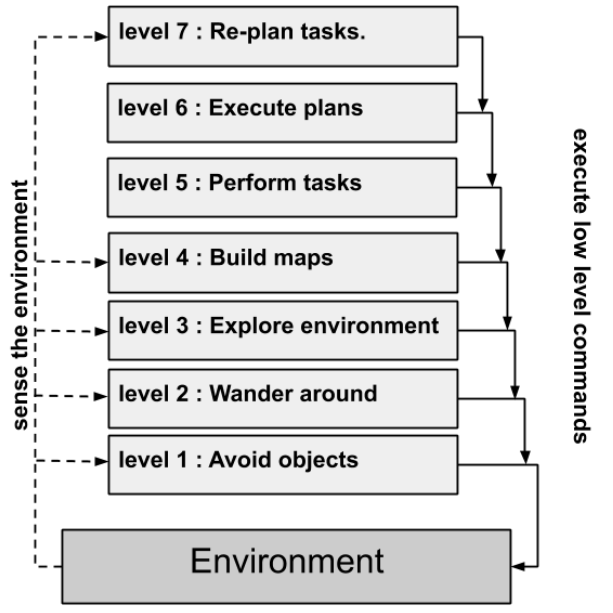

Fig. 5. Subsumption architecture

behaviors [25]. In each layer, it defines a single competence level that performs a specific behavior such as the ability to move away from an obstacle, to move around an area or to explore the robot's environment. It ties together all those increasing layers to build the robot system.

Brooks [24] has defined seven competence levels (Fig. 5), each of which provides a specific autonomy level:

- The first level makes sure that the control system avoids contact with other objects.

- The second level offers the ability to wander around aimlessly without hitting things.

- The third one provides the robot the ability to explore its environment.

- The fourth level builds an appropriate map of the environment and plans the appropriate trajectories to reach some places;

- The fifth level reasons about goals and performs tasks.

- The sixth one executes plans to reach the desired environment.

- The last level reasons about the behavior and appropriately re-plans tasks.

The key idea of the competence level is to provide an easy way to move to the next higher autonomy level. it is reached by adding a new competence level over the existing higher control layer of the whole architecture. [43]:

Subsumption approach presents three major weaknesses

- During execution, the priority-based mechanism limits the ways the system can be adapted.

- Since higher layers interfere with lower ones, they cannot be designed independently.

- Crucial behaviors cannot always be prioritized. 
TABLE I. ANALYSIS OF THE STUDIED CONTROL APPROACHES

\begin{tabular}{l|c|c|c|c|l|l} 
& Deliberative & Reactive & Hybrid & Behavior & $\begin{array}{l}\text { Hybrid } \\
\text { behavior }\end{array}$ & Subsumption \\
\hline Global reasoning & Yes & No & Yes & No & Yes & Yes \\
\hline Reactivity & No & Yes & Yes & Yes & Yes & Yes \\
\hline Adaptability & No & No & No & Yes & Yes & No \\
\hline Flexibility & No & Yes & No & Yes & Yes & Yes \\
\hline Adaptability & No & No & No & Yes & Yes & No \\
\hline Modularity & No & No & No & Yes & No & No \\
\hline Robustness & No & No & No & No & No & Yes \\
\hline Sensor integration & No & No & No & Yes & Yes & No \\
\hline Extensibility & No & No & No & No & No & Yes \\
\hline Reusability & No & No & No & No & No & No \\
\hline
\end{tabular}

TABLE II. DESCRIPTION OF THE SPECIFICATIONS USED IN THE EVALUATION OF THE STUDIED CONTROL APPROACHES

\begin{tabular}{l|l} 
Specification & Description \\
\hline Global reasoning & the architecture makes suitable decisions and preserves the aircraft safety. \\
\hline Reactivity & the architecture recognizes changes and avoids dynamic obstacles. \\
\hline Adaptability & the architecture can carry out a large variety of mission with little reconfiguration requires. \\
\hline Flexibility & the architecture can add new functionality or change the existing one. \\
\hline Modularity & build up the whole architecture using a modular approach. \\
\hline Robustness & the architecture can adapt and repair the task plan when some sensors fail. \\
\hline Sensor integration & the proposed architecture can be adapted to new sensors. \\
\hline Extensibility & the ability to improve the current autonomy level by adding additional modules. \\
\hline Reusability & the same proposed architecture can be used for various UAV type. \\
\hline
\end{tabular}

\section{CONTRol ARChitectures: Comparative Study}

In the preceding section, several contributions in term of robotic control architecture have been described. In a nutshell, we provide the comparative table below (Table I) to summarize the advantages and limitations of each of the studied control strategies. The description of the specifications used in the evaluation of the studied control approaches is listed in Table II.

The comparative study, presented in Table I, showed that the deliberative approach is the more promising control strategy for complex mission, operating in a static environment with complete knowledge of the world model. However, specifically for UAV control system, this approach is neither robust, nor flexible, nor extensible, nor reactive.

The reactive approach represents the best architecture choice for missions that require reactive navigation in a dynamic environment such as UAV target tracking. On the other hand, this approach is ineffective due to its incapability to deal with the high-level goal.

The hybrid control approach enjoys the advantage of meeting missions that need both deliberative and reactive capabilities such as Intelligent Surveillance and Reconnaissance (ISR). However, for UAV system, this control architecture may present a strong drawback, in which the decisive layer has lacks the direct access to the reactive layer; in fact, during task planning, the higher level can have old world model. By this means, the task planner may produce invalid task plan and, consequently, it may fail its overall mission.

Hybrid behavior-based approach presents a good solution for UAV control problem that needs great mission capability, higher flexibility, and adaptability to different mission kind.
Nevertheless, this approach presents two weakness particularly on the UAV system: it is difficult to be implemented and it suffers from the great dependence to sensor system used on the physical robot.

Subsumption architecture offers the ability to build an intelligent and robust UAV control system with the capacity to improve its global autonomy level. However, while this hierarchical architecture depends to the physical system, it's neither modular nor reusable. In addition, due to the layer's interdependency, this approach presents the lack to integrate new sensors on the overall system. Also, it still difficult to be implemented.

\section{CONCLUSION AND FUtURE WORK}

In this paper, we have surveyed different control architecture in attempt to extract the main features that will characterize our autonomous UAV. The comparative study, done in the section three, showed that each approach is more promising in some situations. The deliberative approach can meet complex goals in static environment. The reactive strategy avoids dynamic obstacles. The hybrid architecture combines, in the same architecture, the deliberative and the reactive capabilities. The behavior approach defines a set of modules, each of which can be implemented independently. The hybrid-behavior architecture achieve complex goals by executing independent task. The subsumption approach presents the advantage of improving the autonomy level of the whole architecture.

As conclusion of this work, designing an autonomous UAV for civil applications requires the following features [44]:

- Global reasoning for meeting complex goals [45]. 
- $\quad$ Reactivity to avoid dynamic obstacles [46].

- Adaptability to carry out a wide range of mission.

- $\quad$ Flexibility for adding new functionality [47].

- Modularity for layers independencies [48].

- Robustness to repair the task plan, if needed [24].

- Sensor integration for the capability to add new sensors to the control architecture [24].

- Extensibility for the ability to improve the current autonomy level of the control architecture [49].

- Reusability for the ability to reuse the same architecture in various UAVs [50].

Our future work will be focused on proposing a new control architecture for autonomous UAV operating in civil domain. The proposed architecture will be characterized by the features cited above (see Table II). The proposed architecture must achieve complex goals, perform complicated tasks, compute a feasible trajectory, avoid obstacles and generate an appropriate flight plan.

\section{REFERENCES}

[1] K. P. Valavanis, Advances in Unmanned Aerial Vehicles: State of the Art and the Road to Autonomy. Springer Science \& Business Media, Feb. 2008. Google-Books-ID: EsjPyblwMdQC.

[2] A. Vuruskan, B. Yuksek, U. Ozdemir, A. Yukselen, and G. Inalhan, "Dynamic modeling of a fixed-wing vtol uav," in 2014 International Conference on Unmanned Aircraft Systems (ICUAS), pp. 483-491, IEEE, 2014.

[3] H. Chen, X. Wang, and Y. Li, "A survey of autonomous control for uav," in 2009 International Conference on Artificial Intelligence and Computational Intelligence, vol. 2, pp. 267-271, Nov 2009.

[4] S. G Gupta, M. Ghonge, and P. Jawandhiya, "Review of unmanned aircraft system (uas)," International Journal of Advanced Research in Computer Engineering \& Technology, vol. 9, 042013.

[5] J. D. Boskovic, R. Prasanth, and R. K. Mehra, "A multilayer control architecture for unmanned aerial vehicles," in American Control Conference, 2002. Proceedings of the 2002, vol. 3, pp. 1825-1830, IEEE, 2002.

[6] P. Fahlstrom and T. Gleason, Introduction to UAV systems. John Wiley \& Sons, 2012.

[7] F. Kendoul, "Survey of advances in guidance, navigation, and control of unmanned rotorcraft systems," Journal of Field Robotics, vol. 29, no. 2, pp. 315-378, 2012.

[8] P. Liu, A. Y. Chen, Y.-N. Huang, J.-Y. Han, J.-S. Lai, S.-C. Kang, T.-H Wu, M.-C. Wen, M.-H. Tsai, et al., "A review of rotorcraft unmanned aerial vehicle (uav) developments and applications in civil engineering," Smart Struct. Syst, vol. 13, no. 6, pp. 1065-1094, 2014.

[9] P. Ridao, J. Yuh, J. Batlle, and K. Sugihara, "On AUV control architecture," in Intelligent Robots and Systems, 2000.(IROS 2000). Proceedings. 2000 IEEE/RSJ International Conference on, vol. 2, pp. 855-860, IEEE, 2000.

[10] D. Nakhaeinia, S. H. Tang, S. M. Noor, and O. Motlagh, "A review of control architectures for autonomous navigation of mobile robots," International Journal of Physical Sciences, vol. 6, no. 2, pp. 169-174, 2011.

[11] S. M. Veres, L. Molnar, N. K. Lincoln, and C. P. Morice, "Autonomous vehicle control systems-a review of decision making," Proceedings of the Institution of Mechanical Engineers, Part I: Journal of Systems and Control Engineering, vol. 225, no. 2, pp. 155-195, 2011.

[12] M. Hoy, A. S. Matveev, and A. V. Savkin, "Algorithms for collisionfree navigation of mobile robots in complex cluttered environments: a survey," Robotica, vol. 33, no. 3, pp. 463-497, 2015.
[13] R. G. Simmons, "Structured control for autonomous robots," IEEE transactions on robotics and automation, vol. 10, no. 1, pp. 34-43, 1994.

[14] P. P. Narayan, P. P. Wu, D. A. Campbell, and R. A. Walker, "An intelligent control architecture for unmanned aerial systems (uas) in the national airspace system (nas)," in 2nd International Unmanned Air Vehicle Systems Conference, (Grand Hyatt, Melbourne, Australia), pp. 1-11, 2007.

[15] I. A. Nesnas, A. Wright, M. Bajracharya, R. Simmons, T. Estlin, and W. S. Kim, "CLARAty: An architecture for reusable robotic software," in Unmanned Ground Vehicle Technology V, vol. 5083, pp. 253-265, International Society for Optics and Photonics, 2003.

[16] S. Emel'yanov, D. Makarov, A. I. Panov, and K. Yakovlev, "Multilayer cognitive architecture for uav control," Cognitive Systems Research, vol. 39, pp. 58-72, 2016.

[17] R. Huq, G. K. Mann, and R. G. Gosine, "Mobile robot navigation using motor schema and fuzzy context dependent behavior modulation," Applied Soft Computing, vol. 8, no. 1, pp. 422 - 436, 2008.

[18] J. Pinto, J. Sousa, F. Py, and K. Rajan, "Experiments with deliberative planning on autonomous underwater vehicles," in IROS 2012 Workshop on Robotics for Environmental Monitoring, Vilamoura, Portugal, 2012.

[19] R. C. Arkin, "Motor schema - based mobile robot navigation," The International Journal of Robotics Research, vol. 8, no. 4, pp. 92-112, 1989.

[20] L. Muratet, S. Doncieux, and J.-A. Meyer, "A biomimetic reactive navigation system using the optical flow for a rotary-wing uav in urban environment," Proceedings of the International Session on Robotics, pp. 2262-2270, 2004.

[21] R. Peter Bonasso, R. James Firby, E. Gat, D. Kortenkamp, D. P. Miller, and M. G. Slack, "Experiences with an architecture for intelligent, reactive agents," Journal of Experimental \& Theoretical Artificial Intelligence, vol. 9, pp. 237-256, Apr. 1997.

[22] R. C. Arkin, "Governing lethal behavior: Embedding ethics in a hybrid deliberative/reactive robot architecture," in Proceedings of the 3rd ACM/IEEE international conference on Human robot interaction, pp. 121-128, ACM, 2008.

[23] M. Mataric, "Behavior-based control: Main properties and implications," in In Proceedings, IEEE International Conference on Robotics and Automation, Workshop on Architectures for Intelligent Control Systems, pp. 46-54, 1992.

[24] R. Brooks, "A robust layered control system for a mobile robot," IEEE journal on robotics and automation, vol. 2, no. 1, pp. 14-23, 1986.

[25] E. Oland, T. S. Andersen, and R. Kristiansen, "Subsumption architecture applied to flight control using composite rotations," Automatica, vol. 69, pp. 195-200, 2016.

[26] M. Ghallab, D. Nau, and P. Traverso, Automated planning and acting. Cambridge University Press, 2016.

[27] F. Ingrand and M. Ghallab, "Deliberation for autonomous robots: A survey," Artificial Intelligence, vol. 247, pp. 10-44, 2017.

[28] H.-D. Burkhard, J. Bach, R. Berger, B. Brunswieck, and M. Gollin, "Mental models for robot control," in Advances in Plan-Based Control of Robotic Agents, pp. 71-88, Springer, 2002.

[29] F. R. Noreils and R. G. Chatila, "Plan execution monitoring and control architecture for mobile robots," IEEE Transactions on Robotics and Automation, vol. 11, pp. 255-266, Apr. 1995.

[30] F. Belkhouche, "Reactive optimal uav motion planning in a dynamic world," Robotics and Autonomous Systems, vol. 96, pp. 114-123, 2017.

[31] U. A. Sheikh, M. Jamil, and Y. Ayaz, "A comparison of various robotic control architectures for autonomous navigation of mobile robots," in 2014 International Conference on Robotics and Emerging Allied Technologies in Engineering (iCREATE), pp. 239-243, IEEE, 2014.

[32] K. Nonami, F. Kendoul, S. Suzuki, W. Wang, and D. Nakazawa, Autonomous flying robots: unmanned aerial vehicles and micro aerial vehicles. Springer Science \& Business Media, 2010.

[33] A. D. Ryan, D. L. Nguyen, and J. K. Hedrick, "Hybrid control for uavassisted search and rescue," in ASME 2005 International Mechanical Engineering Congress and Exposition, pp. 187-195, American Society of Mechanical Engineers, 2005 
[34] M. Mataric, "Behavior-Based Control: Main Properties and Implications," in In Proceedings, IEEE International Conference on Robotics and Automation, Workshop on Architectures for Intelligent Control Systems, pp. 46-54, 1992.

[35] H. T. Dinh, M. H. C. Torres, and T. Holvoet, "Sound and complete reactive uav behavior using constraint programming," in ICAPS Workshop on Planning and Robotics, ICAPS Workshop on Planning and Robotics, 2018.

[36] A. Brunete, M. Hernando, E. Gambao, and J. E. Torres, "A behaviour-based control architecture for heterogeneous modular, multiconfigurable, chained micro-robots," Robotics and Autonomous Systems, vol. 60 , no. 12 , pp. 1607-1624, 2012.

[37] M. Carreras, J. Batlle, P. Ridao, and G. Roberts, "An Overview on Behaviour-Based Methods for AUV Control," IFAC Proceedings Volumes, vol. 33, pp. 141-146, Aug. 2000.

[38] L. Iocchi, D. Nardi, and M. Salerno, "Reactivity and deliberation: a survey on multi-robot systems," in Workshop on Balancing Reactivity and Social Deliberation in Multi-Agent Systems, pp. 9-32, Springer, 2000.

[39] J. Liu, H. Hu, and D. Gu, "A Hybrid Control Architecture for Autonomous Robotic Fish," in 2006 IEEE/RSJ International Conference on Intelligent Robots and Systems, pp. 312-317, Oct. 2006.

[40] J.-D. Liu and H. Hu, "Biologically inspired behaviour design for autonomous robotic fish," International Journal of Automation and Computing, vol. 3, no. 4, pp. 336-347, 2006.

[41] M. Mtshali and A. Engelbrecht, "Robotic architectures," Defence Science Journal, vol. 60, no. 1, p. 15, 2010.

[42] G. Butler, A. Gantchev, and P. Grogono, "Object-oriented design of the subsumption architecture," Software: Practice and Experience, vol. 31, no. 9, pp. 911-923, 2001.

[43] R. C. Arkin, An Behavior-based Robotics. Cambridge, MA, USA: MIT Press, 1st ed., 1998.

[44] A. A. Zheltoukhov and L. A. Stankevich, "A survey of control architectures for autonomous mobile robots," in 2017 IEEE Conference of Russian Young Researchers in Electrical and Electronic Engineering (EIConRus), pp. 1094-1099, IEEE, 2017.

[45] C. Sampedro, H. Bavle, J. L. Sanchez-Lopez, R. A. S. Fernández, A. Rodríguez-Ramos, M. Molina, and P. Campoy, "A flexible and dynamic mission planning architecture for uav swarm coordination," in 2016 International Conference on Unmanned Aircraft Systems (ICUAS), pp. 355-363, IEEE, 2016.

[46] A. Viquerat, L. Blackhall, A. Reid, S. Sukkarieh, and G. Brooker, "Reactive collision avoidance for unmanned aerial vehicles using doppler radar," in Field and Service Robotics, pp. 245-254, Springer, 2008.

[47] R. Volpe, I. Nesnas, T. Estlin, D. Mutz, R. Petras, and H. Das, "The CLARAty architecture for robotic autonomy," in Aerospace Conference, 2001, IEEE Proceedings., vol. 1, pp. 1-121, IEEE, 2001.

[48] M. Proetzsch, T. Luksch, and K. Berns, "The behaviour-based control architecture ib2c for complex robotic systems," in Annual Conference on Artificial Intelligence, pp. 494-497, Springer, 2007.

[49] I. A. Nesnas, A. Wright, M. Bajracharya, R. Simmons, T. Estlin, and W. S. Kim, "Claraty: An architecture for reusable robotic software," in Unmanned Ground Vehicle Technology V, vol. 5083, pp. 253-264, International Society for Optics and Photonics, 2003.

[50] S. Joyeux, R. Alami, S. Lacroix, and A. Lampe, "Simulation in the laas architecture," in International Conference on Robotics and AutomationWorkshop on Software Development in Robotics, 2005. 